\title{
Efeitos da oferta de forragem, do método de pastejo, dos dias de avaliação e da raça no comportamento e temperamento de ovinos ${ }^{1}$
}

\section{José Manuel Díaz Gómez ${ }^{2}$, Vivian Fischer ${ }^{3}$, César Henrique Espírito Candal Poli ${ }^{3}$, Paulo César de Faccio Carvalho ${ }^{3}$, Eliezer José Pegoraro' ${ }^{2}$, Stefani Macari²}

\author{
1 Pesquisa financiada pelo CNPq e Agropecuária Cerro Coroado. \\ 2 Programa de pós-graduação em Zootecnia do DZ/FA/UFRGS - Porto Alegre, RS, Brasil. \\ ${ }^{3}$ DZ/FA/UFRGS - Porto Alegre, RS, Brasil.
}

RESUMO - Objetivou-se estudar comportamento e o temperamento de ovinos de três raças (Suffolk, Texel, Ile de France) submetidas a dois métodos de pastejo (contínuo e rotacionado), com duas ofertas de forragem (10 e $20 \mathrm{~kg}$ de MS de pastagem de azevém/100 kg de peso vivo/dia) durante quatro avaliações, entre agosto e novembro de 2006. Utilizou-se o delineamento completamente casualizado em parcelas subsubdivididas, de modo que os métodos de pastejo e as ofertas de forragem constituíram as parcelas, as raças as subparcelas e os dias de avaliação as subsubparcelas. Foram utilizadas 48 fêmeas ovinas com peso médio inicial de 27,59 kg e idade entre 15 e 25 meses. Na avaliação do temperamento, efetuaram-se medidas comportamentais pelo teste de arena, tipo de marcha e tempo de fuga e atributos fisiológicos, como temperatura corporal e frequências cardiorrespiratórias. No método contínuo com baixa oferta de forragem, observou-se maior número de micções. Conforme as avaliações ocorreram, houve aumento da movimentação, das tentativas de fuga, mas menor número de vocalizações durante o isolamento. A raça Ile de France apresentou maior número de tentativas de fuga e de dejeções durante o isolamento. No pastejo rotacionado com alta oferta de forragem, o ganho de peso foi menor. A movimentação no isolamento e na presença do observador foi negativamente relacionada ao ganho de peso. As variáveis descritoras do temperamento apresentaram baixa repetibilidade. O temperamento dos ovinos foi modificado principalmente pelo dia de avaliação e pela raça, ao contrário do sistema de pastejo e da oferta de pasto, embora esses fatores tenham apresentado interação com os dias de avaliação e a raça do animal.

Palavras-chave: distância de fuga, medidas fisiológicas, tempo de fuga, teste de arena

\section{Effect of forage supply, methods of grazing, evaluation days and breed on the behavior and the temperament of sheep}

\begin{abstract}
This work aimed to study the temperament and the behavior of three sheep breeds (Suffolk, Texel, Ile de France) submitted to two grazing methods (continuous and rotational) with two forage supplies (10 and $20 \mathrm{~kg}$ of Italian ryegrass DM/100 kg body weight/day) during four evaluations from August to November 2006. A random block design was used with split-plots, so the grazing methods and forage supplies were the plots, breeds were the sub-plots, and days of evaluation were the sub-sub-plots. It was used 48 ewe lambs with $27.59 \mathrm{~kg}$ of initial weight at 15 to 25 months of age. For evaluation of temperament, behavioral measures were taken by the arena test, and qualitative and quantitative flight time test, and physiological attributes such as body temperature and cardiorespiratory rates. For continuous method with low forage supply, it was observed a higher number of urination events. As evaluations were carried out, physical activity and flight events increased, but number of vocalization decreased during social isolation. Ile de France breed showed higher number of flight attempts and excretion events during social isolation. In the rotational grazing method with high forage supply, weight gain was lower. Physical activity during social isolation and at the observer presence was negatively related to weight gain. Variables used to describe the temperament showed low consistency. The temperament of sheep is mainly affected by evaluation day and breed, unlike grazing method and forage supply, although these factors show interaction with evaluation days and breed.
\end{abstract}

Key Words: arena test, flight distance, flight time, physiological measurements

\section{Introdução}

A ovinocultura no Rio Grande do Sul é conduzida em pastagens nativas extensivamente (Siqueira, 1990) e geralmente apresenta baixa produtividade. Porém, mesmo nos sistemas produtivos mais extensivos, a subdivisão das propriedades e o manejo mais frequente dos animais aumentam o contato destes com os seres humanos, 
promovendo diversos tipos de reações dos animais. Essa reatividade às situações potencialmente estressantes pode ser um fator importante na redução do desempenho e qualidade do produto final (Apple et al., 1995; Moberg, 2000).

O temperamento pode ser definido como a reatividade dos animais frente aos estímulos que provocam o medo (Boissy \& Bouissou, 1995); é influenciado pela sua personalidade (Curley et al., 2004) e depende do manejo prévio e das características genéticas (Le Neindre et al., 1996).

A avaliação do temperamento pode ser realizada pelo teste de arena (Fell \& Shutt, 1989; Kilgour \& SzantarCoddington, 1995), teste do tempo de fuga (Burrow \& Dillon, 1998) ou tipo de marcha (Grandin et al., 1995). Pode ser avaliado com atributos fisiológicos, como temperatura corporal, frequência cardiorrespiratória (Syme \& Elphick, 1982) e cortisol plasmático (Turner, 2004).

Entre os fatores que afetam o temperamento, citam-se a raça (Silveira et al., 2010; Rech et al, 2008), condições de manejo (Silveira et al., 2008a) e dias de avaliação (Silveira et al., 2008b). Embora esses fatores, além da experiência prévia, possam afetar a sua expressão, alguns trabalhos mostram valores de repetibilidade de moderados a altos, ou seja, bovinos muito agitados ou muito calmos tendem a permanecer de tal forma (Grandin, 1993; Roussel et al., 2006; Silveira et al., 2008a).

Objetivou-se com este trabalho avaliar a influência do método de pastejo associado à oferta de forragem, assim como das raças e dos dias de avaliação, sobre o comportamento e o temperamento de cordeiras, e verificar a relação entre o ganho de peso e o temperamento ovino.

\section{Material e Métodos}

O trabalho foi desenvolvido na Estação Experimental Agronômica (EEA) da Universidade Federal do Rio Grande do Sul (UFRGS), situada no km 146 da rodovia BR-290, no município de Eldorado do Sul, com coordenadas geográficas de $30^{\circ} 05^{\prime} 22^{\prime \prime} \mathrm{S}$ de latitude e $51^{\circ} 39^{\prime} 08^{\prime \prime} \mathrm{W}$ de longitude (Bergamaschi \& Guadagni, 1990).
O clima da região é do tipo subtropical úmido, CFA, segundo a classificação de Köeppen (Moreno, 1961), com temperaturas médias entre $14 \mathrm{e} 24^{\circ} \mathrm{C}$, temperaturas máximas e mínimas de 37,3 e $-0,9^{\circ} \mathrm{C}$, podendo ocorrer geadas de abril a outubro. A precipitação média anual é de 1398 mm, sendo as estiagens mais freqüentes em meados de outubro e fins de primavera. Predominam ventos de direção sudeste em todas as estações do ano. As condições meteorológicas observadas nos dias de avaliação do comportamento se situam dentro das faixas de variação médias (Tabela 1).

Foram utilizadas 48 cordeiras com peso médio de 27,59 kg e 15 a 25 meses de idade, raças Ile de France (16), Texel (16) e Suffolk (16), provenientes da Fazenda Angico, município de Cachoeira do Sul, Rio Grande do Sul, pertencentes à Cabanha Cerro Coroado. Os animais foram mantidos em pastagem de azevém (Lolium multiflorum), dividida em 16 piquetes e mantidas em pastejo, com duas ofertas de forragem (10 e $20 \mathrm{~kg}$ de matéria seca/100 kg de peso vivo/dia) em dois métodos de condução de pastejo (lotação contínua e rotacionada.

Foram realizadas quatro avaliações de temperamento nas datas 2/8, 5/9, 2/10 e 23/10/2006, com pesagem prévia dos animais, feita das 8 às $13 \mathrm{~h}$. Na véspera de cada pesagem, os animais foram levados ao curral e mantidos em jejum total por 14 horas. As observações comportamentais do temperamento consistiram da distância de fuga, do teste de arena (Kilgour \& Szantar-Coddington, 1997; Cloete et al., 2005), do tempo de fuga (Burrow \& Dillon, 1998; Silveira et al., 2008b) e do tipo de marcha (Grandin et al., 1995; Silveira et al., 2008b), enquanto as avaliações fisiológicas do temperamento consistiram da mensuração de temperatura corporal e frequências respiratória e cardíaca (Rech, 2006).

As avaliações comportamentais relacionadas ao temperamento foram realizadas segundo três testes: teste de arena e distância de fuga, tempo de fuga e tipo de marcha, descritos a seguir. $\mathrm{O}$ teste de arena e distância de fuga foi realizado num curral coberto, com piso de terra, 9,6 $\mathrm{m}$ de comprimento $\times 8,3 \mathrm{~m}$ de largura e 1,3 $\mathrm{m}$ de altura. Foi

Tabela 1 - Condições meteorológicas nos dias de avaliação do comportamento ${ }^{1}$

\begin{tabular}{|c|c|c|c|c|}
\hline & $2 / 8 / 2006$ & $5 / 9 / 2006$ & $2 / 10 / 2006$ & $23 / 10 / 2006$ \\
\hline Temperatura média $\left({ }^{\circ} \mathrm{C}\right)$ & 7,5 & 6,2 & 19,2 & 20,8 \\
\hline Temperatura máxima $\left({ }^{\circ} \mathrm{C}\right)$ & 14,6 & 13,9 & 26,1 & 28,5 \\
\hline Temperatura mínima $\left({ }^{\circ} \mathrm{C}\right)$ & $-0,3$ & $-1,5$ & 12,2 & 12,1 \\
\hline Umidade relativa do ar (\%) & 79 & 75 & 83 & 73 \\
\hline Precipitação pluviométrica (mm) & 0 & 0 & 0 & 0 \\
\hline Velocidade do vento (m/s) & 1,4 & 1,2 & 1,7 & 1,6 \\
\hline Radiação solar (calc/m²/dia) & 343 & 448 & 449 & 600 \\
\hline
\end{tabular}

${ }^{1}$ Boletim meteorológico (UFRGS, 2006). 
colocada uma lona preta em todo o perímetro para que os animais em teste ficassem isolados visualmente dos companheiros de rebanho. O piso do curral foi dividido em quadrados de $1 \mathrm{~m}^{2}$ com a fixação de cordas ao chão. O teste consistiu de três fases: isolamento, presença humana e tentativa de aproximação. Na primeira fase, cada animal foi colocado isoladamente dentro do curral e observado por 30 segundos quanto à movimentação, tentativas de fuga, número de vocalizações, micções e defecações. A movimentação foi determinada pelo número de quadrados no piso em que os animais colocavam os membros dianteiros. Na segunda fase, o observador entrava e permanecia imóvel, junto ao portão, por mais 30 segundos. Foram observadas as mesmas variáveis da fase anterior. Na terceira fase, o observador tentava se aproximar do animal, interrompendo-se quando o animal se deslocava. A distância de fuga entre o ser humano e o animal foi definida como aquela distância máxima em que o animal permite a aproximação do ser humano sem se deslocar.

O teste de tempo de fuga foi realizado no brete com medidas de 3,0 m de comprimento e 1,3 m de altura, localizado ao lado da mangueira. Avaliou-se o tempo, em segundos, para o animal percorrer 2,0 m, com ajuda de um cronômetro manual (Silveira et al., 2008b). Ao mesmo tempo em que se avaliou o tempo de fuga, outro observador avaliou subjetivamente o tipo de marcha (TM) do animal ao percorrer os $2 \mathrm{~m}$, classificando sua marcha em uma escala de 1 a 6 , em que 1 = caminhada, 2 = caminhada rápida, 3 = trote, 4 = corrida, 5 = resistiu, 6 = pulou (Silveira et al., 2008b).
As características fisiológicas avaliadas foram temperatura corporal medida no reto e frequências cardíaca e respiratória. A temperatura corporal foi medida com termômetro digital colocado no reto no animal durante 1 minuto. As frequências cardíaca e respiratória foram medidas com o estetoscópio durante 1 minuto. As avaliações foram realizadas nas mesmas datas do teste de arena, em curral sem cobertura, começando às $8 \mathrm{~h}$ e terminando às $13 \mathrm{~h}$, em todos os animais, de forma aleatória, de modo a evitar o viés de mensurar os valores de cada sistema de manejo da pastagem, sequencialmente na mesma ordem.

O período experimental foi de 2 de agosto a 12 de novembro de 2006. Em cada piquete, foram colocados animais das três raças. A pastagem foi dividida em 16 piquetes, e cada piquete mediu de 0,23 a 0,41 ha, com uma área total de 4,48 ha, além de uma área de reserva da pastagem. No sistema rotacionado, foram realizados quatro ciclos de pastejo com duração de 31 dias entre o primeiro e o segundo ciclo, 26 dias entre o segundo e o terceiro, 24 dias entre o terceiro e o quarto, e 20 dias entre o quarto e o último ciclo. Os ciclos de pastejo foram predeterminados em combinação com outros experimentos, considerando o período de vida da folha de azevém (Carvalho, 2004) (Tabela 2).

Foi adotado o delineamento experimental completamente casualizado em parcelas subsubdivididas, considerando os métodos de manejo da pastagem $(n=4)$ as parcelas, as raças $(n=3)$ subparcelas e os dias de avaliação $(n=4)$ subsubparcelas.

Tabela 2 - Características da pastagem de azevém anual nos quatro sistemas de manejo e em cada avaliação

\begin{tabular}{|c|c|c|c|c|}
\hline Item & Avaliação 1 & Avaliação 2 & Avaliação 3 & Avaliação 4 \\
\hline \multicolumn{5}{|c|}{ Pastejo contínuo e oferta de $10 \mathrm{~kg}$ MS/100 kg PV/dia } \\
\hline Massa forragem (kg MS/ha) & 1.377 & 1.763 & 3.210 & 1.240 \\
\hline Altura $(\mathrm{cm})$ & 13 & 16 & 16 & 9 \\
\hline Taxa de acúmulo (kg MS/ha/dia) & 46 & 48 & 103 & -38 \\
\hline Oferta de forragem (kg MS/100 kg PV/dia) & 11 & 12 & 17 & 3 \\
\hline \multicolumn{5}{|c|}{ Pastejo contínuo e oferta de $20 \mathrm{~kg}$ MS/100 kg PV/dia } \\
\hline Massa forragem & 1973 & 1953 & 3992 & 2523 \\
\hline Altura & 17 & 26 & 32 & 11 \\
\hline Taxa de acúmulo & 45 & 46 & 123 & -18 \\
\hline Oferta de forragem & 18 & 19 & 44 & 26 \\
\hline \multicolumn{5}{|c|}{ Pastejo rotacionado e oferta de $10 \mathrm{~kg}$ MS/100 kg PV/dia } \\
\hline Massa forragem & 1903 & 2520 & 4098 & 1385 \\
\hline Altura & 22 & 29 & 24 & 8 \\
\hline Taxa de acúmulo & 38 & 35 & 19 & -111 \\
\hline Oferta de forragem & 11 & 12 & 12 & 4 \\
\hline \multicolumn{5}{|c|}{ Pastejo rotacionado e oferta de $20 \mathrm{~kg}$ de MS/100 kg PV/dia } \\
\hline Massa forragem & 2328 & 3263 & 4283 & 2708 \\
\hline Altura & 25 & 36 & 34 & 11 \\
\hline Taxa de acúmulo & 44 & 50 & 37 & -71 \\
\hline Oferta de forragem & 22 & 25 & 24 & 10 \\
\hline
\end{tabular}


Como houve animais que fugiram do curral antes do término do teste de arena, usaram-se os tempos efetivos de permanência dos animais no isolamento (TPI) e durante a presença do observador (TPO) como covariáveis para corrigir as medidas comportamentais, conforme a duração do teste.

A análise estatística das variáveis de natureza contínua e distribuição normal foi realizada por meio da análise da variância, segundo o modelo estatístico: $\mathrm{y}_{\mathrm{ijkl}}=\mathrm{m}+\mathrm{T}_{\mathrm{i}}+$ $\mathrm{P}(\mathrm{T})_{\mathrm{j}(\mathrm{i})}+\mathrm{R}_{\mathrm{k}}+\mathrm{TR}_{\mathrm{ik}}+\mathrm{D}_{\mathrm{l}}+\mathrm{TD}_{\mathrm{il}}+\mathrm{RD}_{\mathrm{kl}}+\mathrm{TPI}+\mathrm{TPO}+\sum_{\mathrm{ijkl}}$, em que, $\mathrm{m}=$ média geral do experimento; $\mathrm{T}_{\mathrm{i}}=$ tratamento $(\mathrm{GL}=3) ; \mathrm{P}(\mathrm{T})_{\mathrm{j}(\mathrm{i})}=$ efeito do piquete aninhado no tratamento $(\mathrm{GL}=12) ; \mathrm{R}_{\mathrm{k}}=$ efeito da raça $(\mathrm{GL}=2) ; \mathrm{TR}_{\mathrm{ik}}=$ efeito da interação tratamento $\times$ raça $(G L=6) ; D_{1}=$ efeito do dia $(\mathrm{GL}=3) ; \mathrm{TD}_{\mathrm{il}}=$ efeito da interação tratamento $\times$ dia $(\mathrm{GL}=9)$; $\mathrm{RD}_{\mathrm{kl}}=$ efeito da interação raça $\times$ dia $(\mathrm{GL}=6)$; TPI = duração efetiva do teste em isolamento; TPO = duração efetiva do teste na presença do observador; $\sum_{\mathrm{ijkl}}=$ erro aleatório. Os tempos de permanência dos animais no isolamento e na presença do observador foram usados como covariáveis, respectivamente, nos atributos medidos durante o isolamento e na presença do observador. Os atributos fisiológicos, tipo de marcha e tempo de fuga e ganho de peso não foram medidos durante a realização do teste de arena, nem ajustados por covariância.

Adotou-se 0,10 como o nível máximo para a probabilidade do erro tipo I. A comparação das médias foi realizada pelo método dos quadrados mínimos, teste de DMS de Fisher. Foi realizada a análise de correlação linear (Pearson) entre ganho de peso e as variáveis comportamentais e fisiológicas. A repetibilidade das medidas descritoras do temperamento foi calculada usando o procedimento MIXED, e dividindo a variância relativa aos animais pela soma da variância composta do resíduo e a dos animais. Foi realizada análise de regressão entre os atributos do comportamento os dias de medida, usando o procedimento REG. A análise estatística das variáveis discretas foi realizada pela análise de frequências e teste de CMH (CochranMantel-Haenzel(SAS, 2001).

\section{Resultados e Discussão}

Os animais em pastejo contínuo, com baixa oferta de forragem, apresentaram os maiores valores para número de micções $(\mathrm{P}=0,0431)$. Os demais atributos do comportamento não variaram entre os sistemas de manejo da pastagem (Tabela 3).

O menor contato com os seres humanos, proporcionado pela lotação contínua em comparação com a rotacionada; e

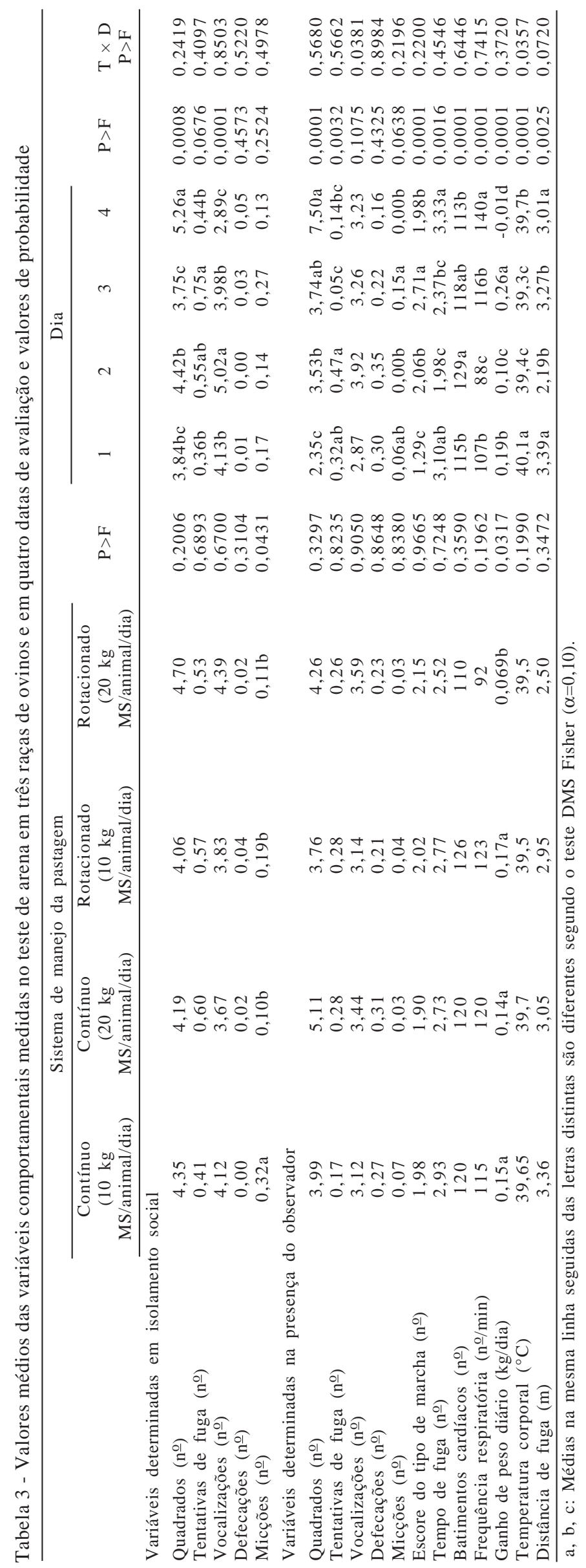

R. Bras. Zootec., v.39, n.8, p.1840-1848, 2010 
a restrição na possibilidade de seleção e apreensão do alimento encontrada na baixa oferta de forragem pode ter influenciado a reação dos cordeiros à manipulação durante as avaliações. O aumento do número de dejeções é costumeiramente aceito como um sinal de estresse (Moberg, 2000; Turner, 2004).

O isolamento social dos companheiros de rebanho e a presença de um componente novo normalmente se constituem em fatores estressantes, que desencadeiam nos animais uma ou mais das seguintes manifestações: aumento da atividade locomotora, aumento das ocorrências de defecação e micção e vocalização (Syme \& Elphyck, 1982; Turner, 2004; Roussel et al., 2006). Porém, em isolamento, os ovinos podem exibir comportamentos distintos, como imobilidade tônica (Syme \& Elphyck, 1982).

O ganho de peso foi menor ( $\mathrm{P}=0,0217$, Tabela 3) no sistema de pastejo rotacionado com alta oferta de forragem, provavelmente porque a pastagem nesse sistema apresentou acúmulo excessivo de forragem (Tabela 2), especialmente de colmos e material senescente, o que pode ter depreciado sua qualidade.

Embora os dados da composição bromatológica da pastagem não estejam disponíveis, pode-se inferir, a partir de resultados obtidos em outro estudo, realizado na mesma área, com pastagem de azevém anual implantada e manejada de forma similar sob o mesmo sistema de manejo (Barbosa et al., 2007), que o sistema rotacionado apresentou menores proporções de lâminas foliares, material morto e percentuais de proteína e digestibilidade, mas maiores percentuais de colmos e celulose em comparação com o sistema contínuo. $\mathrm{Na}$ oferta alta de forragem, o pasto apresentou maior percentual de celulose, mas menor percentual de proteína e digestibilidade que o nível baixo de oferta. Nesse mesmo trabalho, o ganho de peso dos cordeiros foi menor na lotação rotacionada e oferta alta de forragem, comparados respectivamente com a lotação contínua e baixa oferta de forragem.

As variações entre os dias das avaliações abrangeram maior número de atributos que aquelas entre sistemas de manejo da pastagem. A movimentação durante as fases do isolamento e na presença do observador foram maiores na quarta avaliação, o número de fugas durante o isolamento foi maior na terceira avaliação, enquanto o número de vocalizações no isolamento foi menor $(\mathrm{P}<0,10)$ na quarta avaliação comparada com as demais. Esses resultados podem ser relacionados à experiência prévia dos animais ao manejo empregado nas avaliações anteriores, reagindo com maior movimentação dentro da mangueira, mas vocalizando menos.

À medida que ocorreram as avaliações, as variáveis que descreveram a movimentação no teste de arena aumentaram linearmente: $\mathrm{n}^{\circ}$ de tentativas de fugas no isolamento $=-0,08$ $+0,087 \times \operatorname{dia}\left(\mathrm{P}<0,10, \mathrm{R}^{2}=0,03\right)$; movimentação na presença do observador $=0,05+1,68 \times$ dia $\left(P<0,0001, \mathrm{R}^{2}=0,30\right)$; enquanto decresceram o número de vocalizações no isolamento ( $\mathrm{Y}=5,91-0,5 \times$ dia, $\left.\mathrm{P}<0,05 ; \mathrm{R}^{2}=0,05\right)$ e o número de tentativas de fuga na presença do observador $(\mathrm{Y}=0,44$ $-0,11 \times$ dia, $\mathrm{P}<0,05 ; \mathrm{R}^{2}=0,10$ ).

O isolamento social é considerado altamente aversivo para os ovinos, os quais são extremamente gregários. Os ovinos reagiram, inicialmente, vocalizando para localizar os companheiros de rebanho, mas com a experiência adquirida nas avaliações prévias, os animais se movimentaram mais e apresentaram mais tentativas de fuga. Kilgour (1998) observou que os animais diminuíram o número de vocalizações à medida que aumentava a idade e o manejo regular. Pode-se explicar a redução do número de fugas na presença do observador com o passar das avaliações, pois os animais foram mais bem sucedidos nas suas tentativas de fuga e permaneceram menos tempo na mangueira, o que revela o seu aprendizado em evitar uma situação estressante.

O escore do tipo de marcha aumentou no decorrer das avaliações ( $\left.Y=1,21+0,34 \times \operatorname{dia}, P<0,05 ; R^{2}=0,11\right)$, indicando que, inicialmente, os animais caminharam mais devagar. O tempo de fuga do brete foi menor $(\mathrm{P}<0,05)$ nas segunda e na terceira avaliação, condizente com o escore do tipo de marcha. Menores tempos de fuga e maiores escores de tipo de marcha foram associados com maior reatividade dos animais com ovinos (Burrow \& Dillon, 1998) e com bovinos (Silveira et al., 2008a, b). A medida do tipo de marcha não é comumente realizada em ovinos. Entretanto, é bastante usada com bovinos, não exige equipamentos, e valores maiores foram relacionados com maior reatividade (Grandin, 1993).

Todavia, mesmo em bovinos, os resultados quanto à variação das medidas do temperamento entre avaliações é contraditório. Existem trabalhos que relatam pequena variação entre avaliações, indicando consistência (Barbosa Silveira, 2005; Grandin et al., 1995). Em outros estudos, ocorreu aumento na movimentação, possivelmente modulado pela experiência prévia da exposição a situações percebidas como estressantes pelos animais (Petherick et al., 2002; Müller \& Keyserlingk, 2006); enquanto outros autores verificaram a diminuição na movimentação dos animais, denotando a habituação desses ao manejo (King et al., 2006).

O número de batimentos cardíacos foi maior na segunda avaliação, diminuindo na última avaliação. Com o decorrer das avaliações, a frequência respiratória aumentou $\left(\mathrm{Y}=84,03+10,72 \times \operatorname{dia}, \mathrm{P}<0,05 ; \mathrm{R}^{2}=0,11\right)$. Animais submetidos ao manejo mais frequente e ao maior contato com pessoas podem apresentar menos estresse que os 
animais manejados raramente (Fordyce et al., 1988; Rushen et al., 2001), todavia o aumento da temperatura ambiente e da radiação solar constatado nas duas últimas avaliações (Tabela 1) pode ter influenciado a frequência respiratória.

O ganho de peso diário foi menor na ultima avaliação comparada com as demais (Tabela 3), provavelmente influenciado pela redução da quantidade (Tabela 2) e qualidade da pastagem, esta estimada pela diminuição da possibilidade de seleção, explicado pelo estádio reprodutivo e por resultados de composição bromatológica e botânica de outros trabalhos na mesma área (Barbosa et al., 2007).

Houve interações significativas entre sistemas de manejo da pastagem e dias de avaliação para os atributos: número de vocalizações na presença do observador, distância de fuga e temperatura corporal (Tabela 3).

Os ovinos mantidos em pastejo contínuo e alta oferta de forragem vocalizaram mais na presença do observador na terceira avaliação, enquanto no pastejo rotacionado e alta oferta de pasto vocalizaram menos na segunda avaliação, não diferindo nas demais avaliações entre os sistemas de manejo da pastagem. Os animais do sistema de pastejo pastejo contínuo com baixa oferta de forragem apresentaram menor distância de fuga na primeira avaliação, mas maior valor deste atributo na terceira avaliação em comparação aos demais. Os animais mantidos em pastejo rotacionado nos dois níveis de oferta de pasto e no pastejo contínuo e alta oferta de pasto apresentaram maior valor de temperatura corporal quando contidos na primeira avaliação em relação às demais avaliações, enquanto os animais mantidos no pastejo contínuo e baixa oferta de pasto apresentaram maiores valores na primeira e na última avaliação.

Aparentemente os animais mantidos no pastejo contínuo e baixa oferta de pasto reagiram mais intensamente nas últimas avaliações. Possivelmente o menor contato com os humanos e a maior restrição alimentar tenham influenciado a suscetibilidade dos animais ao estresse, provocando a sensitização dos animais, ou seja, o aumento da manifestação do comportamento com o passar das avaliações.

As cordeiras Ile de France mostraram comportamentos mais ativos, exibindo maior número de tentativas de fuga e eventos de defecação durante o isolamento que as cordeiras da raça Suffolk. As cordeiras Ile de France também apresentaram maior movimentação na presença do observador que as demais, quando mantidas em pastejo contínuo e alta oferta de pasto, e maior distância de fuga que as cordeiras Suffolk, quando mantidas em pastejo rotacionado com alta oferta de forragem. Cordeiras Texel apresentaram maior frequência respiratória $(\mathrm{P}<0,05)$ que cordeiras das outras raças na segunda e terceira avaliação. As cordeiras Suffolk vocalizaram mais durante o isolamento e na presença do observador que as cordeiras Ile de France e Texel quando mantidas em pastejo contínuo com alta oferta de forragem (Tabela 4). As cordeiras Ile de France mostraram maior reatividade no teste de arena, enquanto as cordeiras Texel mostraram maior reatividade de acordo com os atributos fisiológicos.

Tabela 4 - Probabilidades de rejeição da hipótese de nulidade dos efeitos de raça, dia de avaliação e sua interação e valores médios das variáveis comportamentais medidas no teste de arena de ovinos mantidos em pastagem de azevém

\begin{tabular}{|c|c|c|c|c|c|c|c|c|}
\hline & \multicolumn{3}{|c|}{ Raça } & \multirow{2}{*}{$\frac{\text { Raça }}{\mathrm{P}>\mathrm{F}}$} & \multirow{2}{*}{$\frac{\text { Raça } \times \text { trat }}{\mathrm{P}>\mathrm{F}}$} & \multirow{2}{*}{$\frac{\text { Raça } \times \text { dia }}{\mathrm{P}>\mathrm{F}}$} & \multirow[t]{2}{*}{$\mathrm{R}^{2}$ modelo } & \multirow[t]{2}{*}{ CV (\%) } \\
\hline & Ile de France & Suffolk & Texel & & & & & \\
\hline \multicolumn{9}{|c|}{ Variáveis determinadas em isolamento social } \\
\hline Vocalizações (nํㅜ) & 3,83 & 4,34 & 3,85 & 0,3413 & 0,0294 & 0,4109 & 0,53 & 53,5 \\
\hline Defecações (no$)$ & $0,07 \mathrm{a}$ & $0,0 \mathrm{~b}$ & $0,0 \mathrm{~b}$ & 0,0062 & 0,5069 & 0,6218 & 0,22 & 680,6 \\
\hline Micções (noํ) & 0,16 & 0,17 & 0,21 & 0,7577 & 0,6771 & 0,9324 & 0,27 & 207,5 \\
\hline Quadrados ( $\left.\mathrm{n}^{\circ} \underline{\mathrm{o}}\right)$ & $5,21 \mathrm{a}$ & $3,69 b$ & $3,94 b$ & 0,0347 & 0,0737 & 0,0934 & 0,58 & 62,9 \\
\hline Tentativas de fuga ( $\mathrm{n}^{\mathrm{O}}$ ) & 0,21 & 0,31 & 0,22 & 0,5536 & 0,5433 & 0,5969 & 0,44 & 187,7 \\
\hline Vocalizações $\left(\mathrm{n}^{\mathrm{o}}\right)$ & 3,00 & 3,71 & 3,25 & 0,2196 & 0,0996 & 0,1229 & 0,50 & 52,6 \\
\hline Micções (n무) & 0,16 & 0,32 & 0,28 & 0,3844 & 0,9794 & 0,6543 & 0,26 & 1725,6 \\
\hline Defecações $(n \underline{0})$ & 0,00 & 0,04 & 0,08 & 0,4962 & 0,1579 & 0,9913 & 0,25 & 392,1 \\
\hline Temperatura corporal $\left({ }^{\circ} \mathrm{C}\right)$ & 39,7 & 39,7 & 39,6 & 0,6909 & 0,2563 & 0,1663 & 0,51 & 1,18 \\
\hline Ganho de peso diário (kg/dia) & 0,17 & 0,14 & 0,13 & 0,4963 & 0,4963 & 0,4816 & 0,47 & 102,9 \\
\hline
\end{tabular}

a, b, c: Médias na mesma linha seguidas das letras distintas são diferentes segundo o teste DMS Fisher $(\alpha=0,10)$. 
Os presentes resultados não estão de acordo com os de Silveira et al. (2010). Esses autores avaliaram animais das raças Corriedale, Ideal, Texel e Suffolk em pista de remate quanto à agitação na entrada e permanência em pista de remate. Os animais das raças Texel e Suffolk mostraram-se mais agitados do que aqueles das raças Corriedale e Ideal, mas não apresentaram diferenças entre si.

Rech et al. (2008) avaliaram ovelhas das raças Ideal e Corriedale e verificaram que estas últimas foram mais reativas e apresentaram maior escore para o tipo de marcha.

Foram detectadas correlações lineares moderadas e positivas $(r<0,50, P<0,05)$ entre a movimentação no teste de arena e o número de vocalizações medidas, respectivamente, no isolamento e na presença do observador, indicando que, nesses atributos, os animais reagiram de forma semelhante quando isolados socialmente e na presença do observador.

O número de vocalizações durante o isolamento foi negativamente relacionado com o número de tentativas de fuga durante o isolamento $(\mathrm{r}=-0,29, \mathrm{P}<0,05)$, com o número de defecações no isolamento $(\mathrm{r}=-0,17, \mathrm{P}<0,05)$, com a freqüência respiratória ( $\mathrm{r}=-0,32, \mathrm{P}<0,05)$, enquanto o número de vocalizações na presença do observador foi negativamente relacionado com a freqüência respiratória $(r=-0,21$, $\mathrm{P}<0,05)$ e com a temperatura corporal $(\mathrm{r}=-0,23, \mathrm{P}<0,05)$. A vocalização parece ter sido mais usada para comunicação entre os animais e tentativa de localização dos companheiros de rebanho.

A movimentação no isolamento foi positivamente relacionada com a frequência respiratória, enquanto a movimentação na presença do observador foi positivamente relacionada com o número de eventos de defecação ( $r=0,30$, $\mathrm{P}<0,05)$, frequência respiratória $(\mathrm{r}=0,33, \mathrm{P}<0,05)$. O número de tentativas de fuga durante o isolamento foi positivamente relacionado com o número de eventos de defecação ( $\mathrm{r}=0,20$, $\mathrm{P}<0,05)$ e frequência cardíaca $(\mathrm{r}=0,16, \mathrm{P}<0,05)$, indicando que os animais mais agitados também apresentaram maiores valores do outros atributos relacionados com o estresse.

Todavia os coeficientes de correlação foram baixos a moderados, indicando que diferentes testes podem exercer desafios distintos, provocando respostas diferentes dos animais, como no isolamento social, na presença humana e contenção física (Silveira et al., 2008b; Rech, 2006).

O ganho de peso diário das cordeiras foi negativamente relacionado com a movimentação dos animais no isolamento $(r=-0,18, P<0,05)$ e na presença do observador $(r=-0,29$, $\mathrm{P}<0,05)$. Em bovinos, maiores ganhos de peso foram observados em animais menos agitados (Voisinet et al., 1997a; Müller \& Keyserlingk, 2006), provavelmente devido ao maior consumo de alimentos (Brown et al., 2004) ou à melhor conversão alimentar (Petherick et al., 2002).
Ao contrário, Rech (2006) não constatou relação entre as variáveis descritoras do temperamento, exceto para cortisol plasmático, com ganho de peso e qualidade de carne. Todavia verificou que a maior suscetibilidade dos animais ao estresse, muitas vezes expressa como maior agitação, foi relacionada negativamente com menor ganho de peso de bovinos, e associada com menor consumo e mais tempo gasto com vigília (Silveira et al., 2008b; King et al, 2006; Muller \& Keyserlingk, 2006) e perdas em aspectos reprodutivos em ovinos (Murphy et al., 1994).

A expressão do comportamento no teste de arena reflete a integração de vários fatores motivacionais: medo do ser humano, motivação social, curiosidade e pode variar conforme os indivíduos. Embora a agitação dos animais seja comumente associada ao estresse, pode ocorrer que a motivação dos animais seja outra, como no estudo realizado por Beausoleil et al. (2008). Na comparação entre os animais com extremos de movimentação (pouca $\times$ muita) no teste de arena, aqueles com elevada atividade locomotora apresentaram baixo nível de medo dos humanos e grande motivação social (reencontro com coespecíficos), mas relação negativa com o nível de cortisol plasmático. Segundo os mesmos autores, o medo pode ser expresso como comportamento de evitação, relutância em investigar o estímulo (objeto, ser humano) e a supressão de comportamentos ativos como locomoção, vocalização e exploração.

Os animais podem perceber diferentemente as práticas de manejo conforme suas experiências anteriores e suscetibilidade ao estresse. A organização da resposta ao agente estressor não é apenas função da percepção do referido agente, mas também de experiências passadas, o que permite o reconhecimento de uma dimensão psicológica do estresse. A capacidade de planejar ou antecipar um evento futuro são processos cognitivos relevantes na gestão de situações potencialmente promotoras de sofrimento (Chandroo et al., 2004), o que explica parcialmente as diferenças observadas entre as quatro avaliações.

A repetibilidade das medidas descritoras do temperamento comportamentais e fisiológicas foi baixa, com destaque para o número de tentativas de fuga no isolamento e na presença do observador $(0,27$ e 0,13$)$, número de quadrados percorridos em isolamento $(0,14)$, número de vocalizações no isolamento e na presença do observador $(0,34$ e 0,19$)$. Os demais valores foram mais baixos. Esse resultado indica que a avaliação do temperamento dos ovinos não deve ser realizada uma única vez, pois os valores se modificaram no decorrer das avaliações.

Esses valores são inferiores aos reportados por outros autores como Kilgour (1998), Reale \& Festa-Bianchet (2003) e Beausoleil et al. (2008) e indicam maior efeito do ambiente 
do que genético sobre a manifestação dessas características no presente estudo, além de grande variação individual (Boissy \& Bouissou, 1995); Beausoleil et al., 2008). Outro fator que pode ter influenciado os resultados foi o intervalo de tempo entre as medidas, em torno de 28 dias, no presente trabalho. Nos trabalhos referenciados, esse intervalo foi superior, em muitos meses, provavelmente permitindo a maior expressão genética das características.

Wolf et al. (2008) trabalharam com ovelhas cruzadas com Bluefaced Leicester e relataram variações nos valores de repetibilidade de $0,58-0,71 ; 0,38-0,40$ e $0,17-0,60$, respectivamente, para o número de vocalizações, número de quadrados percorridos e aproximação dos humanos, sem detectar efeito significativo da fazenda de origem ou entre sexos. Kilgour (1998) encontrou valores médios a altos para a repetibilidade para o número de vocalizações $(0,25)$ e número de quadrados percorridos $(0,61)$ em ovelhas e carneiros da raça Merina, enquanto Reale \& Festa-Bianchet (2003)verificaram consistência dos atributos (impetuosidade x timidez e docilidade) em ovelhas da raça Big Horn.

Esses resultados também são distintos dos de Silveira et al. (2008a), que encontraram valores altos de repetibilidade, superiores a 0,40, para as medidas tempo de fuga, velocidade de saída, distância de fuga, para bovinos de corte, criados em três sistemas de produção.

\section{Conclusões}

O método de pastejo e a oferta de forragem afetam pouco o temperamento dos animais, sobretudo no sistema de pastejo rotacionado, por permitir maior contato com o homem, reduz-se a reatividade dos animais, especialmente nas últimas avaliações. Com o passar das avaliações, a reatividade dos animais aumenta, mediada pela experiência prévia. As raças diferem quanto ao comportamento, e a raça Ile de France reage mais ativamente ao isolamento. Animais mais agitados no teste de arena apresentam menor ganho de peso. O estudo do impacto do temperamento de ovinos sobre o seu ganho de peso dever ser realizado empregandose as variáveis que descrevem a agitação ou movimentação dos animais. Dada a sua baixa repetibilidade, o temperamento de ovinos não deve ser definido por uma única avaliação.

\section{Referências}

APPLE, J.K; DIKEMAN, M.E.; MINTON, J.E. et al. Effects of restrain and isolation stress and epidural blockade on endocrine and blood metabolite status, muscle glycogen metabolism, and indice of dark-cutting longissimus muscle of Sheep. Journal of Animal Science, v.73, p.2295-2307, 1995.

BARBOSA, C.M.P.; CARVALHO, P.C.F.; CAUDURO, G.F. et al. Terminação de cordeiros em pastagens de azevém anual manejadas em diferentes intensidades e métodos de pastejo. Revista Brasileira de Zootecnia, v.36, n.6, p.1953-1960, 2007 (supl).

BARBOSA SILVEIRA, I.D. Influência de genética bovina na susceptibilidade ao estresse durante o manejo e seus efeitos na qualidade da carne. 2005. 180f. Tese (Doutorado em Ciências) - Universidade Federal de Pelotas, Pelotas.

BEAUSOLEIL, N.J.; BLACHE, D.; STAFFORD, K. et al. Exploring the basis of divergent selection for temperament in domestic sheep. Applied Animal Behavior Science, v.109, n.1-2, p.261-274, 2008.

BERGAMASCHI, H.; GUADAGNI, H.R. Agroclima da Estação Experimental Agronômica/UFRGS. Porto Alegre: Departamento de Plantas Forrageiras e Agrometeorologia da UFRGS, 1990. $60 \mathrm{p}$.

BOISSY, A.; BOUISSOU, M.F. Assessment of individual differences in behavioural reactions of heifers exposed to various feareliciting situations. Applied Animal Behaviour Science, v.46, n.1-2, p.17-31, 1995

BURROW, H.M.; DILLON, R.D. Relationships between temperament and growth in a feedlot and commercial carcass traits of bos indicus crossbreds. Australian Journal of Experimental Agriculture, v.37, n.4, p.407-411, 1998.

CARVALHO, P.C.F. Manejando pastagens para ovinos. In: NETO, O.A.P. (Org.). Práticas em ovinocultura: ferramentas para o sucesso. Porto Alegre: SENAR, 2004. p.136.

CHANDROO, K.P.; DUNCAN, I.J.H.; MOCCIA, R.D. Can fish suffer? Perspectives on sentiense, pain, fear and stress. Applied Animal Behavior Science, v.86, p.225-250, 2004.

CLOETE, J.J.E.; CLOETE, S.W.P.; HOFFMAN, L.C. Behaviour of Merinos divergently selected for multiple rearing ability in response to external stimuli. Small Ruminant Research, v.60, n.3, p.227-236, 2005.

CURLEY, K.O.J.; NEUENDORFF, D.A.; LEWIS, A.W. et al. Evaluation of temperament and stress physiology may be useful in breeding programs. In: DEPARTMENT OF ANIMAL SCIENCES. Beef cattle research in Texas publication, section physiology. Maryland: College Station Texas A\&M University, 2004. 265p.

FELL, L.R.; SHUTT, D.A. Behavioural and hormonal responses to acute surgical stress in sheep. Applied Animal Behaviour Science, v.22, n.3-4, p.283-294, 1989

FORDYCE, G.; DODT, R.M.; WYTHES, J.R. Cattle temperaments in extensive beef herds in northern Queensland. 1. Factors affecting temperament. Australian Journal of Experimental Agriculture, v.28, n.6, p.683-687, 1988.

GRANDIN, T. Behavioural agitation during handling of cattle is persistent over time. Applied Animal Behaviour Science, v.36, n.1, p.1-9, 1993.

GRANDIN, T.; DEESING, M.J.; STRUTHERS, J.J. et al. Cattle with hair whore patterns above the ayes are more behaviorally agitated during restraint. Applied Animal Behaviour Science, v.46, n.1, p.117-123, 1995.

KILGOUR, R.J.; SZANTAR-CODDINGTON, M.R. Arena behaviour of ewes selected for superior mothering ability differs from that of unselected ewes. Animal Reproduction Science, v.37, n.2, p.133-141, 1995.

KILGOUR, R.J.; SZANTAR-CODDINGTON, M.R. The arena test and cortisol response of sheep as indirect selection criteria for the improvement of lamb survival. Animal Reproduction Science, v.46, n.1-2, p.97-108, 1997.

KILGOUR, R.J. Arena behaviour is a possible selection criterion for lamb-rearing ability; it can be measured in young rams and ewes. Applied Animal Behaviour Science, v.57, n.1-2, p.81-89, 1998.

KING D.A.; PFEIFFER, C.E.S.; RANDEL, R.D. et al. Influence of animal temperament and stress responsiveness on the carcass quality and beef tenderness of feedlot cattle. Meat Science, v.74, p.546-556, 2006 
LE NEINDRE, P.; BOIVIN, X.; BOISSY, A. Handling of extensively kept animals. Applied Animal Behaviour Science, v.49, n.1, p.73-81, 1996.

MOBERG, G.P. Biological response to stress: implicationsfor animal welfare. In: MOBERG, G.P.; MENCH, J.A. (Eds). The biology of animal stress: basic principles and implications for animal welfare. Davis: CABI Publishing, 2000. p.1-22.

MÜLLER, R.; VON KEYSERLINGK, M.A.G. Consistency of flight speed and its correlation to productivity and to personality in Bos taurus beef cattle. Applied Animal Behavior Science, v.99, p.193-204, 2006.

MURPHY, P.M.; PURVIS, I.W.; LINDSAY, D.R. et al. Measures of temperament are highly repeatable in Merino sheep and some are related to maternal behaviour. In: BIENNIAL CONFERENCE OF THE AUSTRALIAN SOCIETY OF ANIMAL PRODUCTION, 20., 1994, Perth. Proceedings... Perth: ASAP, 1994. 250p.

PETHERICK, J.C.; HOLROYD, R.G.; DOOGAN, V.J. et al. Productivity, carcass and meat quality of lot fed Bos indicus cross steers grouped according to temperament. Australian Journal of Experimental Agriculture, v.42, n.4, p.389-398, 2002.

REALE, D.; FESTA-BIANCHET, M. Predator-induced natural selection on temperament in bighorn ewes. Animal Behaviour, v.65, n.3, p.463-470, 2003.

RECH, C.L.S. Relação entre temperamento, desempenho animal e qualidade de carne em ovinos. Pelotas: UFPEL, 2006. 117f. Tese (Doutorado em Ciências) - Universidade Federal de Pelotas, Pelotas.

RECH, C.L.S.; RECH, J.L.; FISCHER, V. et al. Temperamento e comportamento materno-filial de ovinos das raças Corriedale e Ideal e sua relação com a sobrevivência dos cordeiros. Ciência Rural, v.38, n.5, p.1388-1393, 2008.

ROUSSÉL, S.; HEMSWORTH, P.; LERUSTE, H. et al. Repeated transport and isolation during pregnancy in ewes: Effects on the reactivity to humans and to their offspring after lambing. Applied Animal Behaviour Science, v.97, n.2-4, p.172-189, 2006.
RUSHEN, J.; MUNKSGAARD, L.; MARNET, P.G. et al. Human contact and the effects of acute stress on cows at milking. Applied Animal Behaviour Science, v.73, n.1, p.1-14, 2001.

SILVEIRA, I.D.B.; FISCHER, V.; WIEGAND, M.M. Temperamento de bovinos de corte: métodos de medida em diferentes sistemas produtivos. Archivos de Zootechnia, v.57, n.219, p.321-332, 2008a.

SILVEIRA, I.D.B.; FISCHER, V.; RESTLE, J. Relação entre diferentes genótipos de novilhos confinados Charolês x Nelore e temperamento. Revista Brasileira de Zootecnia, v.37, n.10, p.1808-1814, 2008b.

SILVEIRA, I.D.B.; FISCHER, V.; MENDONÇA, G. Efeito do genótipo e idade de ovinos na reatividade medida em pista de venda. Revista Brasileira de Zootecnia, 2010 (prelo).

SIQUEIRA, E.R. Raças ovinas e sistemas de produção. In: SILVA SOBRINHO, A.G. (Ed.) Produção de ovinos. Jaboticabal: FUNEP, 1990. 211p.

STATISTICAL ANALYSES SYSTEM - SAS. SAS/STAT ${ }^{\circledR}$ User's guide. Version 8.1, 6.ed. Cary: SAS Institute Inc., v.2, 2001. 846p.

SYME, L.A.; ELPHICK, G.R. Heart rate and the behaviour of sheep in yards. Applied Animal Ethology, v.9, n.1, p.31-35, 1982.

TURNER, J. Silent lambs: A report on sheep welfare in Scotland. Edinburg: Advocates for Animals, 2004. 50p.

VOISINET, B.D.; GRANDIN, T.; TATUM, J.D. et al. Feedlot cattle with calm temperaments have higher average daily gains than cattle with excitable temperaments. Journal of Animal Science, v.75, p.892-896. 1997a.

UNIVERSIDADE FEDERAL DO RIO GRANDE DO SUL - UFRGS Boletim Agrometereológico. Eldorado do Sul: Estação Experimental Agronômica/UFRGS; Porto Alegre: Faculdade de Agronomia, 2006. (CD-ROM).

WOLF, B.T.; McBRIDE, S.D.; LEWIS, R.M. et al. Estimates of the genetic parameters and repeatability of the behavioural traits of sheep in an arena test. Applied Animal Behaviour Science, v.112, p.68-80, 2008. 